\title{
ALGEBRAIC INDEPENDENCE RESULTS RELATED TO PATTERN SEQUENCES IN DISTINCT $\langle q, r\rangle$-NUMERATION SYSTEMS
}

\author{
YoHeI TACHIYA
}

(Received June 1, 2011, revised November 17, 2011)

\begin{abstract}
In this paper, we prove the algebraic independence over $\boldsymbol{C}(z)$ of the generating functions of pattern sequences defined in distinct $\langle q, r\rangle$-numeration systems. Our result asserts that any nontrivial linear combination over $\boldsymbol{C}$ of pattern sequences chosen from distinct $\langle q, r\rangle$-numeration systems can not be a linear recurrence sequence. As an application, we give a linear independence over $\boldsymbol{C}$ of the pattern sequences.
\end{abstract}

1. Introduction and main results. Let $q \geq 2$ and $r$ be fixed integers with $r \in$ $\{0,1, \ldots, q-2\}$. Any positive integer $n$ is uniquely expressed as

$$
n=\sum_{i=0}^{k} a_{i} q^{i}, \quad a_{i} \in \Sigma_{q, r}, \quad a_{k}>0,
$$

for an integer $k \geq 0$, where $\Sigma_{q, r}:=\{-r, 1-r, \ldots, 0,1, \ldots, q-1-r\} \supset\{0,1\}$. The set of all finite nonempty strings of elements in $\Sigma_{q, r}$ is denoted by $\Sigma_{q, r}^{*}$. Then the string of $\langle q, r\rangle$-digits

$$
(n)_{q, r}:=a_{k} \cdots a_{1} a_{0} \in \Sigma_{q, r}^{*}
$$

is called the $\langle q, r\rangle$-expansion of $n$. The $\langle q, 0\rangle$-expansion is the ordinary $q$-ary expansion. These numeration systems are called $\langle q, r\rangle$-numeration systems.

For $w \in \Sigma_{q, r}^{*}$, we write $w^{k}=w w \cdots w$ (k times). In particular $w^{0}$ denotes the empty word. If $w \neq 0^{l}$ for any $l \geq 1$, we say that $w$ is a nonzero pattern. For a nonzero pattern $w \in \Sigma_{q, r}^{*}$, we define $e_{q, r}(w ; 0)=0$ and $e_{q, r}(w ; n)(n \geq 1)$ to be the number of (possibly overlapping) occurrences of $w$ in the $\langle q, r\rangle$-expansion of $n$. Here, in evaluating $e_{q, r}(w ; n)$ for $n \geq 1$, we suppose that the $\langle q, r\rangle$-expansion of $n$ has an arbitrary long string of zeros on the left. The resulting sequence $\left\{e_{q, r}(w ; n)\right\}_{n \geq 0}$ is called the pattern sequence for the pattern $w$ in the $\langle q, r\rangle$-numeration system (cf. Allouche and Shallit [1]).

Define the generating function of the pattern sequence for $w \in \Sigma_{q, r}^{*}$ by

$$
f(w ; z):=\sum_{n \geq 0} e_{q, r}(w ; n) z^{n} .
$$

For any fixed integer $q \geq 2$ and any patterns $w_{1}, \ldots, w_{m} \in \Sigma_{q, 0}^{*}$, Uchida [10] gave necessary and sufficient conditions for the generating functions $f\left(w_{1} ; z\right), \ldots, f\left(w_{m} ; z\right)$ to be

2000 Mathematics Subject Classification. Primary 11J85; Secondary 11A63, 11J72.

Key words and phrases. Mahler type functional equation, algebraic independence, pattern sequences, $\langle q, r\rangle$ numeration systems. 
algebraically dependent over $\boldsymbol{C}(z)$. Shiokawa and the author [7, 8] generalized this results to any fixed $\langle q, r\rangle$-numeration systems and studied the linear relations between the pattern sequences. Recently, the author [9] proved that the generating functions of pattern sequences defined in distinct $q$-ary number systems are algebraically independent over $\boldsymbol{C}(z)$.

In this paper, we prove the algebraic independence over $\boldsymbol{C}(z)$ of the generating functions of pattern sequences defined in distinct $\langle q, r\rangle$-numeration systems. The tools to prove the following main theorem are also similar to those in [9], however we need some different techniques.

THEOREM 1.1. Let $w_{q, r} \in \Sigma_{q, r}^{*}(q=2,3, \ldots, r=0,1, \ldots, q-2)$ be any nonzero patterns. Then the functions

$$
f_{q, r}(z)=\sum_{n \geq 0} e_{q, r}\left(w_{q, r} ; n\right) z^{n} \quad(q=2,3, \ldots, r=0,1, \ldots, q-2)
$$

are algebraically independent over $\boldsymbol{C}(z)$. Furthermore, the values $f_{q, r}(\alpha)(q=2,3, \ldots$, $r=0,1, \ldots, q-2)$ are algebraically independent over $\boldsymbol{Q}$ for any algebraic number $\alpha$ with $0<|\alpha|<1$.

Applying Theorem 1.1, we see that any nontrivial linear combination over $\boldsymbol{C}$ of the functions (1) cannot be a rational function, namely,

$$
\sum_{q=2}^{m} \sum_{r=0}^{q-2} c_{q, r} f_{q, r}(z) \notin \boldsymbol{C}(z)
$$

for an arbitrary integer $m \geq 2$ and $c_{q, r} \in \boldsymbol{C}$ not all zero. Hence we have the following corollary.

COROllary 1.2. Let $w_{q, r} \in \Sigma_{q, r}^{*}(q=2,3, \ldots, r=0,1, \ldots, q-2)$ be any nonzero patterns and $c_{q, r} \in \boldsymbol{C}$ not all zero. Then the linear combination of the pattern sequences

$$
\left\{\sum_{q=2}^{m} \sum_{r=0}^{q-2} c_{q, r} e_{q, r}\left(w_{q, r} ; n\right)\right\}_{n \geq 0}
$$

cannot be a linear recurrence sequence. In particular, the sequences $\left\{e_{q, r}\left(w_{q, r} ; n\right)\right\}_{n \geq 0}(q=$ $2,3, \ldots, r=0,1, \ldots, q-2)$ are linearly independent over $\boldsymbol{C}$.

EXAMPLE 1.3. Let $w=a_{k} \cdots a_{0}$ be a nonzero pattern with $a_{i} \in\{0,1\}$. Then $e_{2,0}(w ; n)$ and $e_{3,1}(w ; n)$ indicate the number of occurrences of $w$ in the binary and the balanced ternary expansions of $n$, respectively, and any nontrivial linear combination over $\boldsymbol{C}$

$$
\left\{c_{1} e_{2,0}(w ; n)+c_{2} e_{3,1}(w ; n)\right\}_{n \geq 0}
$$

cannot be a linear recurrence sequence. 
EXAMPLE 1.4. For any integer $q \geq 2$, the pattern sequences

$$
\begin{gathered}
\left\{e_{2,0}(1 ; n)\right\}_{n \geq 0},\left\{e_{3,0}(1 ; n)\right\}_{n \geq 0},\left\{e_{3,1}(1 ; n)\right\}_{n \geq 0}, \ldots, \\
\left\{e_{q, 0}(1 ; n)\right\}_{n \geq 0}, \ldots,\left\{e_{q, q-2}(1 ; n)\right\}_{n \geq 0},
\end{gathered}
$$

which are defined by the number of "1" appearing in each $\langle q, r\rangle$-numeration systems of $n$, are linearly independent over $\boldsymbol{C}$.

2. Some properties of pattern sequences. In this section, we show as Proposition 2.1 a non-periodicity for the linear combination of the pattern sequences defined in distinct $\left\langle q^{i}, r_{i}\right\rangle$-numeration systems $(i=1, \ldots, m)$. Proposition 2.1 will be used in the proof of Theorem 1.1 (see Section 4).

PROPOSITION 2.1. Let $q \geq 2$ be a fixed integer, $m \geq 1$ be any integer, and $w_{q^{i}, r_{i}} \in$ $\Sigma_{q^{i}, r_{i}}^{*}\left(i=1,2, \ldots, m, r_{i} \in\left\{0,1, \ldots, q^{i}-2\right\}\right)$ be arbitrary nonzero patterns. Then for any $c_{i} \in \boldsymbol{C}(i=1, \ldots, m)$, not all zero, the sequence

$$
\left\{c_{1} e_{q, r_{1}}\left(w_{q, r_{1}} ; n\right)+c_{2} e_{q^{2}, r_{2}}\left(w_{q^{2}, r_{2}} ; n\right)+\cdots+c_{m} e_{q^{m}, r_{m}}\left(w_{q^{m}, r_{m}} ; n\right)\right\}_{n \geq 0}
$$

cannot be purely periodic with a period $q^{l}(l \geq 1)$.

To prove Proposition 2.1, we need some lemmas. In what follows, let $q \geq 2$ and $r \in$ $\{0,1, \ldots, q-2\}$ be fixed integers. For any nonzero pattern $w=b_{l-1} b_{l-2} \cdots b_{0} \in \Sigma_{q, r}^{*}$ with $b_{i} \in \Sigma_{q, r}$, let $|w|$ denote the length $l$ and put

$$
v(w):=\left\{\begin{array}{cl}
\sum_{k=0}^{l-1} b_{k} q^{k} & \text { if } \sum_{k=0}^{l-1} b_{k} q^{k}>0, \\
q^{l}+\sum_{k=0}^{l-1} b_{k} q^{k} & \text { otherwise. }
\end{array}\right.
$$

By definition, $0<v(w)<q^{l}$. The following Lemma 2.2 is a generalization of [9, Lemma 1] to the case of $\langle q, r\rangle$-numeration systems with $r=1,2, \ldots, q-2$.

LEMMA 2.2. Let $m \geq 1$ and $d \geq 0$ be integers. Then for any nonzero pattern $w \in$ $\Sigma_{q^{m}, r}^{*}$ with $w \neq 0^{l} 1(l \geq 0)$, we have

$$
e_{q^{m}, r}\left(w ; v(w) q^{d}\right)= \begin{cases}1 & \text { if } d \equiv 0(\bmod m) \\ 0 & \text { otherwise }\end{cases}
$$

PROOF. In the case of $r=0$, the equality (2) holds for all nonzero patterns $w \in \Sigma_{q^{m}, 0}^{*}$ (cf. [9, Lemma 1]). Let $r \geq 1$ and

$$
w=0^{l_{1}} a_{k} \cdots a_{0} 0^{l_{2}}, \quad a_{j} \in \Sigma_{q^{m}, r}, \quad a_{k}, a_{0} \neq 0, \quad l_{1}, l_{2} \geq 0 .
$$

Then we have

$$
(v(w))_{q^{m}, r}=\left\{\begin{aligned}
a_{k} \cdots a_{0} 0^{l_{2}} & \text { if } a_{k}>0, \\
10^{l_{1}} a_{k} \cdots a_{0} 0^{l_{2}} & \text { otherwise } .
\end{aligned}\right.
$$

If $d$ is divisible by $m$, noting that $a_{k}, a_{0} \neq 0$, we see that the pattern $w$ appears just once in the $\left\langle q^{m}, r\right\rangle$-expansion of $v(w) q^{d}$. Hence the equality (2) holds for this case. In what follows, we 
assume that $d$ is not divisible by $m$ and put $d=m h+u$, where $h \geq 0$ and $u(1 \leq u \leq m-1)$ are integers.

Here we prove (2) with assuming $a_{k}>0$. The case $a_{k}<0$ is proved similarly. Let

$$
\left(v(w) q^{u}\right)_{q^{m}, r}=b_{s} b_{s-1} \cdots b_{0} 0^{l_{2}} \in \Sigma_{q^{m}, r}^{*}, \quad b_{j} \in \Sigma_{q^{m}, r}, \quad b_{s}>0 .
$$

Then we have $\left(v(w) q^{d}\right)_{q^{m}, r}=\left(v(w) q^{u}\left(q^{m}\right)^{h}\right)_{q^{m}, r}=b_{s} b_{s-1} \cdots b_{0} 0^{l_{2}+h}$ with $s=k$ or $k+1$, since

$$
k+1+l_{2}=\left|(v(w))_{q^{m}, r}\right| \leq\left|\left(v(w) q^{u}\right)_{q^{m}, r}\right| \leq\left|\left(v(w) q^{m}\right)_{q^{m}, r}\right|=k+2+l_{2} .
$$

Suppose on the contrary that $e_{q^{m}, r}\left(w ; v(w) q^{d}\right) \geq 1$, that is, the pattern $w$ appears at least once in the expansion (4). If $s=k$, then $w=0^{l_{1}} b_{k} b_{k-1} \cdots b_{0} 0^{l_{2}}$, which implies $v(w)=v(w) q^{u}$ from (4). This is impossible, and hence $s=k+1$. We distinguish the two cases.

Case I. $l_{2}=0$. If $l_{1} \geq 1$, noting that $b_{k+1} \neq 0$, we see that the pattern $w=0^{l_{1}} a_{k} \cdots a_{0}$ coincides with $0^{l_{1}} b_{k+1} b_{k} \cdots b_{1}$. Hence we obtain by (4)

$$
v(w) q^{m}+b_{0}=v\left(b_{k+1} \cdots b_{1}\right) q^{m}+b_{0}=v\left(b_{k+1} \cdots b_{1} b_{0}\right)=v(w) q^{u},
$$

so that

$$
0<v(w)=\frac{-b_{0}}{q^{m}-q^{u}} \leq \frac{q^{m}-2}{q^{m}-q^{u}}<2,
$$

which implies $v(w)=1$, namely $w=0^{l_{1}} 1$. By the assumption, this is a contradiction.

Suppose that $l_{1}=0$. Then the pattern $w$ must be of the form either $w=b_{k+1} \cdots b_{1}$ or $w=b_{k} \cdots b_{0}$. In a similar way to the case $l_{1} \geq 1$, we can show $w \neq b_{k+1} \cdots b_{1}$. If $w=b_{k} \cdots b_{0}$, then

$$
v(w)+b_{k+1}\left(q^{m}\right)^{k+1}=v\left(b_{k} \cdots b_{0}\right)+b_{k+1}\left(q^{m}\right)^{k+1}=v\left(b_{k+1} \cdots b_{1} b_{0}\right)=v(w) q^{u},
$$

so that $b_{k+1} q^{m(k+1)}=v(w)\left(q^{u}-1\right)$. Since $u \geq 1$, the positive integers $q^{m(k+1)}$ and $q^{u}-1$ are coprime, and hence $v(w) \equiv 0\left(\bmod q^{m(k+1)}\right)$. This implies $a_{j}=0$ for all $j=0, \ldots, k$ in (3), a contradiction.

Case II. $l_{2} \geq 1$. In the case of $l_{1} \geq 1$, the pattern $w$ must be the form $w=0^{l_{1}} b_{k+1} \ldots$ $b_{1} b_{0} 0^{l_{2}-1}$ with $b_{0}=0$. Hence we have by (4)

$$
v(w)=v\left(b_{k+1} \cdots b_{1} b_{0} 0^{l_{2}-1}\right)=v(w) q^{u-m},
$$

a contradiction.

If $l_{1}=0$, then $w=b_{k+1} \cdots b_{1} b_{0} 0^{l_{2}-1}\left(b_{0}=0\right)$ or $w=b_{k} \cdots b_{0} 0^{l_{2}}$. By the same way as above, we can deduce $w \neq b_{k+1} \cdots b_{1} b_{0} 0^{l_{2}-1}\left(b_{0}=0\right)$. Furthermore, we see that $w \neq b_{k} \cdots b_{0} 0^{l_{2}}$ by a similar way to the proof of Case I.

For the patterns $w=0^{l} 1 \in \Sigma_{q^{m}, r}^{*}(l \geq 0)$, we prepare the following lemma;

LEMMA 2.3. Let $w \in \Sigma_{q^{m}, r}^{*}$ be of the form $w=0^{l} 1$ for some $l \geq 0$. Then for any integer $k \geq 1$, we have

$$
e_{q^{m}, r}\left(w ; v\left(w^{k}\right) q^{d}\right)= \begin{cases}k & \text { if } d \equiv 0(\bmod m) \\ 0 \text { or } 1 & \text { otherwise }\end{cases}
$$


Proof. The assertion is trivial for the case that $d$ is divisible by $m$. Suppose that $d$ is not divisible by $m$ and put $d=m h+u$, where $h \geq 0$ and $u(1 \leq u \leq m-1)$ are integers. If $q^{u} \in \Sigma_{q^{m}, r}$, we have

$$
\left(v\left(w^{k}\right) q^{d}\right)_{q^{m}, r}=\left(v\left(w^{k}\right) q^{u}\left(q^{m}\right)^{h}\right)_{q^{m}, r}=q^{u}\left(0^{l} q^{u}\right)^{k-1} 0^{h},
$$

so that $e_{q^{m}, r}\left(w ; v\left(w^{k}\right) q^{d}\right)=0$. If $q^{u} \notin \Sigma_{q^{m}, r}$, then $b:=-\left(q^{m}-q^{u}\right) \in \Sigma_{q^{m}, r}$ and $\left(q^{u}\right)_{q^{m}, r}=$ $1 b \in \Sigma_{q^{m}, r}^{*}$. Noting that $b<0$, we have for $w=0^{l} 1$

$$
\left(v\left(w^{k}\right) q^{d}\right) q^{m, r}= \begin{cases}1(b+1)^{k-1} b 0^{h} & \text { if } l=0, \\ 1 b\left(0^{l-1} 1 b\right)^{k-1} 0^{h} & \text { otherwise, }\end{cases}
$$

where $b, b+1 \in \Sigma_{q^{m}, r} \backslash\{1\}$. Hence $e_{q^{m}, r}\left(w ; v\left(w^{k}\right) q^{d}\right)=1$ and the lemma is proved.

Let $m \geq 2$ be an integer. Define

$$
S_{m-1}:=\left\{\left(k_{1}, k_{2}, \ldots, k_{m-1}\right) \in \boldsymbol{Z}^{m-1} ; 0 \leq k_{j} \leq j-1\right\}
$$

and its subset

$$
S_{m-1, n}:=\left\{\left(k_{1}, k_{2}, \ldots, k_{m-1}\right) \in S_{m-1} ; k_{1}+\cdots+k_{m-1}=n\right\}
$$

for each $n=0,1, \ldots,(m-1)(m-2) / 2$. Note that $S_{m-1}=\bigcup_{n \geq 0} S_{m-1, n}$.

LEMMA 2.4 (cf. [9, Lemma 2]). For any integer $m \geq 2$, there exist integers $d_{1}$ and $d_{2}$ with $0 \leq d_{1}<d_{2} \leq m-1$ such that

$$
\sum_{\substack{n \geq 0 \\ n \equiv d_{1}(\bmod m)}} \sharp S_{m-1, n} \neq \sum_{\substack{n \geq 0 \\ n \equiv d_{2}(\bmod m)}} \sharp S_{m-1, n},
$$

where $\sharp S_{m-1, n}$ is the number of elements in $S_{m-1, n}$.

Proof of Proposition 2.1. We prove the lemma by induction on $m$. The assertion is trivial for $m=1$. Indeed, for any pattern $w \in \Sigma_{q, r}^{*}$ and the integer $n_{j}:=v\left(w^{j}\right)>0$, we have $e_{q, r}\left(w ; n_{j}\right) \rightarrow \infty$ as $j \rightarrow \infty$. Let $m \geq 2$ and assume the assertion for lower $m$. Suppose on the contrary that there exist $c_{i} \in \boldsymbol{C}$ not all zero such that the sequence

$$
\left\{c_{1} e_{q, r_{1}}\left(w_{q, r_{1}} ; n\right)+c_{2} e_{q^{2}, r_{2}}\left(w_{q^{2}, r_{2}} ; n\right)+\cdots+c_{m} e_{q^{m}, r_{m}}\left(w_{q^{m}, r_{m}} ; n\right)\right\}_{n \geq 0}
$$

is purely periodic with a period $q^{l}$ for some $l \geq 1$. We may assume $c_{m} \neq 0$. Let $d_{1}$ and $d_{2}$ $\left(0 \leq d_{1}<d_{2} \leq m-1\right)$ be integers as in Lemma 2.4 and

$$
N_{j}=\sum_{k_{1}=0}^{0} \sum_{k_{2}=0}^{1} \cdots \sum_{k_{m-1}=0}^{m-2} q^{k_{1}+\cdots+k_{m-1}-d_{j}+D L l\left(1+k_{1}+m k_{2}+\cdots+m^{m-2} k_{m-1}\right)}, \quad j=1,2,
$$

where $D:=\operatorname{lcm}(1,2, \ldots, m-1)$ and $L$ is a sufficiently large integer. Since $N_{1}, N_{2} \equiv 0$ $\left(\bmod q^{l}\right)$ and the sequence $(7)$ is periodic with the period $q^{l}$, we get

$$
\sum_{i=1}^{m} c_{i} e_{q^{i}, r_{i}}\left(w_{q^{i}, r_{i}} ; a N_{1}\right)=\sum_{i=1}^{m} c_{i} e_{q^{i}, r_{i}}\left(w_{q^{i}, r_{i}} ; a N_{2}\right)
$$


for any integer $a>0$. On the other hand, in a similar way to the proof of [9, Theorem 1], we can prove

$$
e_{q^{i}, r_{i}}\left(w_{q^{i}, r_{i}} ; a N_{1}\right)=e_{q^{i}, r_{i}}\left(w_{q^{i}, r_{i}} ; a N_{2}\right), \quad i=1,2, \ldots, m-1 .
$$

Hence, combining (8) and (9), we obtain for any integer $a>0$

$$
e_{q^{m}, r_{m}}\left(w_{q^{m}, r_{m}} ; a N_{1}\right)-e_{q^{m}, r_{m}}\left(w_{q^{m}, r_{m}} ; a N_{2}\right)=0 .
$$

In what follows, we show the existence of the integer $a>0$ satisfying

$$
e_{q^{m}, r_{m}}\left(w_{q^{m}, r_{m}} ; a N_{1}\right) \neq e_{q^{m}, r_{m}}\left(w_{q^{m}, r_{m}} ; a N_{2}\right)
$$

and deduce a contradiction. We distinguish two cases.

Case I. $w_{q^{m}, r_{m}} \neq 0^{l} 1$ for any $l \geq 0$. For the integer $a=v\left(w_{q^{m}, r_{m}}\right)$, we have by Lemma 2.2

$$
\begin{aligned}
e_{q^{m}, r_{m}}\left(w_{q^{m}, r_{m}} ; a N_{j}\right) & =\sum_{k_{1}=0}^{0} \ldots \sum_{k_{m-1}=0}^{m-2} e_{q^{m}, r_{m}}\left(w_{q^{m}, r_{m}} ; a q^{k_{1}+\cdots+k_{m-1}+m-d_{j}+D L}\right) \\
& =\sharp\left\{\left(k_{1}, \ldots, k_{m-1}\right) \in S_{m-1} ; \sum_{i=1}^{m-1} k_{i} \equiv d_{j}(\bmod m)\right\} \\
& =\sum_{\substack{n \geq 0 \\
n \equiv d_{j}(\bmod m)}} \sharp S_{m-1, n}, \quad j=1,2,
\end{aligned}
$$

where $S_{m-1}$ and $S_{m-1, n}$ are the finite sets defined by (5) and (6), respectively. Hence we obtain (10) by Lemma 2.4 .

Case II. $w_{q^{m}, r_{m}}=0^{l} 1$ for some $l \geq 0$. We may assume

$$
\sum_{\substack{n \geq 0 \\ n \equiv d_{1}(\bmod m)}} \sharp S_{m-1, n}<\sum_{\substack{n \geq 0 \\ n \equiv d_{2}(\bmod m)}} \sharp S_{m-1, n} .
$$

Let $a=v\left(w_{q^{m}, r_{m}}^{m !}\right)$. Then we have

$$
e_{q^{m}, r_{m}}\left(w_{q^{m}, r_{m}} ; a N_{1}\right)=m ! \sum_{\substack{n \geq 0 \\ n \equiv d_{1}(\bmod m)}} \sharp S_{m-1, n}+\sum_{\substack{n \geq 0 \\ n \neq d_{1}(\bmod m)}} \delta_{n} \sharp S_{m-1, n},
$$

where $\delta_{n}:=e_{q^{m}, r_{m}}\left(w_{q^{m}, r_{m}} ; a q^{n-d_{1}+D L}\right)$. By Lemma $2.3, \delta_{n}=0$ or 1 for each $n \geq 0$. Hence, noting that

$$
\sum_{\substack{n \geq 0 \\ n \neq d_{1}(\bmod m)}} \sharp S_{m-1, n} \leq \sum_{n \geq 0} \sharp S_{m-1, n}=\sharp S_{m-1}<m !,
$$

we obtain the inequalities

$$
e_{q^{m}, r_{m}}\left(w_{q^{m}, r_{m}} ; a N_{1}\right)<m ! \sum_{\substack{n \geq 0 \\ n \equiv d_{2}(\bmod m)}} \sharp S_{m-1, n} \leq e_{q^{m}, r_{m}}\left(w_{q^{m}, r_{m}} ; a N_{2}\right),
$$

which implies (10). Therefore Proposition 2.1 is proved. 
3. Lemmas for the proof of Theorem 1.1. We prepare some lemmas for proving Theorem 1.1. In the next section, we show Theorem 1.1 by using these lemmas together with Proposition 2.1.

LEMMA 3.1 (Nishioka [6, Corollary]). Let $K$ be an algebraic number field and $d_{1}, \ldots, d_{t} \geq 2$ be integers with $\log d_{i} / \log d_{j} \notin Q$ if $i \neq j$. Suppose that $f_{i, j}(z) \in K[[z]]$ $(i=1, \ldots, t, j=1, \ldots, m(i))$ satisfy the functional equations

$$
f_{i, j}(z)=f_{i, j}\left(z^{d_{i}}\right)+b_{i, j}(z), \quad b_{i, j}(z) \in K(z) .
$$

If for each $i, f_{i, 1}(z), \ldots, f_{i, m(i)}(z)$ are algebraically independent over $K(z)$ and all $f_{i, j}(z)$ converge at an algebraic number $z=\alpha$ with $0<|\alpha|<1$, then the values

$$
f_{i, j}(\alpha) \quad(i=1, \ldots, t, \quad j=1, \ldots, m(i))
$$

are algebraically independent over $\boldsymbol{Q}$.

Lemma 3.2 (Kubota [2], Loxton and van der Poorten [3]; see also Nishioka [5, Theorem 3.2.1]). Let $d \geq 2$ be an integer. Suppose that $g_{1}(z), \ldots, g_{m}(z) \in \boldsymbol{C}[[z]]$ are algebraically dependent over $\boldsymbol{C}(z)$ and satisfy the functional equations

$$
g_{i}\left(z^{d}\right)=g_{i}(z)+a_{i}(z) \quad a_{i}(z) \in \boldsymbol{C}(z), \quad i=1,2, \ldots, m .
$$

Then there exist constants $c_{1}, \ldots, c_{m} \in \boldsymbol{C}$ not all zero such that

$$
c_{1} g_{1}(z)+c_{2} g_{2}(z)+\cdots+c_{m} g_{m}(z) \in \boldsymbol{C}(z) .
$$

The following lemma is a generalization of [7, Lemma 3], which is the case $L=d^{2}-1$ of the lemma.

LEMMA 3.3. Let $d \geq 2, l \geq 1$, and $L \geq 1$ be arbitrary integers. Suppose that $c(z) \in \boldsymbol{C}(z)$ satisfies the functional equation

$$
c\left(z^{d}\right)=c(z)+\frac{1-z^{L}}{1-z^{d^{l} L}} a(z), \quad a(z) \in \boldsymbol{C}[z] .
$$

Then there exists $b(z) \in \boldsymbol{C}[z]$ such that

$$
c(z)=\frac{1-z^{L}}{1-z^{d^{l-1} L}} b(z)
$$

with $\operatorname{deg} b(z) \leq-L+d^{-1} \max \left\{d^{l} L, L+\operatorname{deg} a(z)\right\}$. Here we understand $\operatorname{deg} 0=-\infty$.

Proof. Similarly to the proof of [7, Lemma 3], we see that there exists $h(z) \in C[z]$ such that $c(z)=h(z) /\left(1-z^{d^{l-1} L}\right)$ and

$$
h\left(z^{d}\right)=\left(1+z^{d^{l-1} L}+z^{2 d^{l-1} L}+\cdots+z^{(d-1) d^{l-1} L}\right) h(z)+\left(1-z^{L}\right) a(z) .
$$

Let $\zeta$ be an arbitrary $L$-th root of unity. Substituting $z=\zeta^{d^{i}}(i=1,2, \ldots)$ into the above identity, we have $h\left(\zeta^{d^{i+1}}\right)=d h\left(\zeta^{d^{i}}\right)$, so that $h\left(\zeta^{d^{i}}\right)=d^{i} h(\zeta)$ for every $i \geq 1$. If $h(\zeta) \neq 0$, then $\left|h\left(\zeta^{d^{i}}\right)\right| \rightarrow \infty$ as $i \rightarrow \infty$, a contradiction. Hence $b(z)=h(z) /\left(1-z^{L}\right) \in \boldsymbol{C}[z]$ and 
we obtain the form (13). Next we estimate the degree of $b(z)$. Substituting $c(z)$ in (13) to the equation (12) and multiplying the both sides by $\left(1-z^{d^{l} L}\right) /\left(1-z^{L}\right)$, we have

$$
\frac{1-z^{d^{l} L}}{1-z^{d^{l-1} L}} b(z)=\frac{1-z^{d L}}{1-z^{L}} b\left(z^{d}\right)-a(z) .
$$

If the degree of the first term of the right-hand side is not greater than that of the left-hand side, we get $\operatorname{deg} b(z) \leq\left(d^{l-1}-1\right) L$. Otherwise, the degree of the first term coincides with $\operatorname{deg} a(z)$, so that $\operatorname{deg} b(z)=-L+(\operatorname{deg} a(z)+L) / d$. The lemma is proved.

4. Proof of Theorem 1.1. Let $N$ be the set of positive integers and $M$ be a subset of $N$ defined by

$$
M=\left\{q \in N ; q \neq a^{n} \quad \text { for any } a, n \in N, n \geq 2\right\} .
$$

Then $\log q_{1} / \log q_{2} \notin \boldsymbol{Q}$ for distinct integers $q_{1}, q_{2} \in M$ and

$$
N \backslash\{1\}=\bigcup_{q \in M}\left\{q, q^{2}, \ldots\right\} \quad \text { (disjoint union) } .
$$

We can choose distinct integers $q_{1}, \ldots, q_{t} \in M$ such that the set of nonzero patterns

$$
w_{i, j, r} \in \Sigma_{q_{i}^{j}, r}^{*} \quad\left(i=1,2, \ldots, t, \quad j=1,2, \ldots, m(i), \quad r=0,1, \ldots, q_{i}^{j}-2\right)
$$

includes the nonzero patterns given in Theorem 1.1. Let

$$
f_{i, j, r}(z)=\sum_{n \geq 0} e_{q_{i}^{j}, r}\left(w_{i, j, r} ; n\right) z^{n}
$$

$\left(i=1, \ldots, t, j=1, \ldots, m_{i}, r=0, \ldots, q_{i}^{j}-2\right)$. By [7, Lemma 5], the functions (14) satisfy Mahler type functional equations

$$
f_{i, j, r}(z)=\frac{1-z^{q_{i}^{j}}}{z^{r}(1-z)} f_{i, j, r}\left(z^{q_{i}^{j}}\right)+\frac{z^{v\left(w_{i, j, r}\right)}}{1-z^{q_{i}^{j\left|w_{i, j, r}\right|}}} .
$$

We transform the functions $f_{i, j, r}(z)$ into the functions which satisfy the functional equation (11). Let $L$ and $u_{i, j, r}$ be positive integers defined by $L=\prod_{i=1}^{t} \prod_{j=1}^{m(i)}\left(q_{i}^{j}-1\right)$ and $u_{i, j, r}=$ $r L /\left(q_{i}^{j}-1\right)$, respectively. Multiplying the both sides of (15) by $z^{r}(1-z)$ and substituting $z^{L}$ for $z$, we get

$$
z^{r L}\left(1-z^{L}\right) f_{i, j, r}\left(z^{L}\right)=\left(1-z^{q_{i}^{j} L}\right) f_{i, j, r}\left(z^{q_{i}^{j} L}\right)+\frac{z^{\left(r+v\left(w_{i, j, r}\right)\right) L}\left(1-z^{L}\right)}{1-z^{q_{i}^{j\left|w_{i, j, r}\right|} L}} .
$$

Noting that $r L=-u_{i, j, r}+q_{i}^{j} u_{i, j, r}$, we obtain the functional equation

$$
F_{i, j, r}(z)=F_{i, j, r}\left(z^{q_{i}^{j}}\right)+\frac{z^{v\left(w_{i, j, r}\right) L-u_{i, j, r}}\left(1-z^{L}\right)}{1-z^{q_{i}^{j\left|w_{i, j, r}\right|} L}},
$$

where

$$
F_{i, j, r}(z):=z^{-u_{i, j, r}}\left(1-z^{L}\right) f_{i, j, r}\left(z^{L}\right) \in Q[[z]] .
$$


Using (16) repeatedly, we have

$$
F_{i, j, r}(z)=F_{i, j, r}\left(z^{q_{i}^{D_{i}}}\right)+\sum_{k=0}^{D_{i} / j-1} z^{q_{i}^{k j}\left(\nu\left(w_{i, j, r}\right) L-u_{i, j, r}\right)} \frac{1-z^{q_{i}^{k j} L}}{1-z^{q_{i}^{j\left|w_{i, j, r}\right|+k j} L}},
$$

where $D_{i}=\operatorname{lcm}(1,2, \ldots, m(i))$.

Proof of TheOREM 1.1. Suppose on the contrary that the first assertion of Theorem 1.1 does not holds, namely, the functions $f_{i, j, r}(z)(i=1, \ldots, t, j=1, \ldots, m(i), \quad r=$ $\left.0, \ldots, q^{i}-2\right)$ defined by (14) are algebraically dependent over $\boldsymbol{C}(z)$. Then so are the functions $F_{i, j, r}(z)$, and hence the values $F_{i, j, r}(\alpha)\left(i=1, \ldots, t, j=1, \ldots, m(i), \quad r=0, \ldots, q^{i}-2\right)$ are algebraically dependent over $\boldsymbol{Q}$ for any algebraic number $\alpha$ with $0<|\alpha|<1$ (cf. Nesterenko [4, Lemma 2.3]). Therefore by Lemma 3.1 together with (16), the functions $F_{i, j, r}(z)\left(j=1, \ldots, m(i), \quad r=0, \ldots, q^{i}-2\right)$ are algebraically dependent over $\boldsymbol{Q}(z)$ for some fixed $i(1 \leq i \leq t)$.

Thus, for a fixed integer $q \geq 2$ we may assume that the functions

$$
F_{j, r}(z)=z^{-u_{j, r}}\left(1-z^{L}\right) \sum_{n \geq 0} e_{q^{j}, r}\left(w_{j, r} ; n\right) z^{L n}, \quad w_{j, r} \in \Sigma_{q^{j}, r}^{*},
$$

$\left(j=1, \ldots, m, \quad r=0,1, \ldots, q^{j}-2\right)$ are algebraically dependent over $\boldsymbol{Q}(z)$, where $u_{j, r}:=$ $r L /\left(q^{j}-1\right)$. By $(18)$

$$
F_{j, r}(z)=F_{j, r}\left(z^{q^{D}}\right)+\sum_{k=0}^{D / j-1} z^{q^{k j}\left(L v\left(w_{j, r}\right)-u_{j, r}\right)} \frac{1-z^{q^{k j} L}}{1-z^{q^{j\left|w_{j, r}\right|+k j} L}},
$$

with $D:=\operatorname{lcm}\{1,2, \ldots, m\}$, and by Lemma 3.2 there exist constants $c_{j, r} \in \boldsymbol{C}$ not all zero such that

$$
R(z):=\sum_{j=1}^{m} \sum_{r=0}^{q^{j}-2} c_{j, r} F_{j, r}(z) \in C(z) .
$$

Without loss of generality, we may assume $c_{m, r_{m}} \neq 0$ for some $r_{m}\left(0 \leq r_{m} \leq q^{m}-2\right)$. Using (19) we obtain

$$
\begin{aligned}
R(z) & =R\left(z^{q^{D}}\right)+\sum_{j=1}^{m} \sum_{r=0}^{q^{j}-2} \sum_{k=0}^{D / j-1} c_{j, r} z^{q^{k j}\left(L v\left(w_{j, r}\right)-u_{j, r}\right)} \frac{1-z^{q^{k j} L}}{1-z^{q^{\left|w_{j, r}\right| j+k j} L}} \\
& =R\left(z^{q^{D}}\right)+\frac{1-z^{L}}{1-z^{q^{D l} L}} a(z),
\end{aligned}
$$

where $l:=\max \left\{\left|w_{j, r}\right| ; 1 \leq j \leq m, 0 \leq r \leq q^{j}-2\right\}$ and

$$
a(z)=\sum_{j=1}^{m} \sum_{r=0}^{q^{j}-2} \sum_{k=0}^{D / j-1} c_{j, r} z^{q^{k j}\left(L v\left(w_{j, r}\right)-u_{j, r}\right)} \frac{1-z^{q^{k j} L}}{1-z^{L}} \frac{1-z^{q^{D l} L}}{1-z^{q^{\left|w_{j, r}\right| j+k j} L}} .
$$


Since $\left|w_{j, r}\right| j+k j \leq\left(\left|w_{j, r}\right|+D / j-1\right) j \leq D l$, we get $a(z) \in C[z]$ with $\operatorname{deg} a(z) \leq$ $\left(q^{D l}-1\right) L$ if $a(z) \neq 0$. Hence, applying Lemma 3.3 with $d=q^{D}$ and $c(z)=R(z) \in C(z)$, we see that there exists $b(z) \in C[z]$ such that

$$
R(z)=\frac{1-z^{L}}{1-z^{q^{D(l-1)} L}} b(z),
$$

where $\operatorname{deg} b(z) \leq\left(q^{D(l-1)}-1\right) L$ if $b(z) \neq 0$. By the expression (20) and the definition of $R(z)$, we have

$$
\begin{aligned}
b(z) & =\frac{1-z^{q^{D(l-1)} L}}{1-z^{L}} \sum_{j=1}^{m} \sum_{r=0}^{q^{j}-2} c_{j, r} F_{j, r}(z) \\
& =\left(1-z^{q^{D(l-1)} L}\right) \sum_{n \geq 0} \sum_{j=1}^{m} \sum_{r=0}^{q^{j}-2} c_{j, r} e_{q^{j}, r}\left(w_{j, r} ; n\right) z^{n L-u_{j, r}} \\
& =Q(z)+\sum_{n \geq 0} \sum_{j=1}^{m} \sum_{r=0}^{q^{j}-2}\left(d_{j, r}\left(n+q^{D(l-1)}\right)-d_{j, r}(n)\right) z^{n L+q^{D(l-1)} L-u_{j, r}},
\end{aligned}
$$

where $d_{j, r}(n):=c_{j, r} e_{q^{j}, r}\left(w_{j, r} ; n\right)$ and

$$
Q(z):=\sum_{n=0}^{q^{D(l-1)}-1} \sum_{j=1}^{m} \sum_{r=0}^{q^{j}-2} d_{j, r}(n) z^{n L-u_{j, r}} \in \boldsymbol{C}[z] .
$$

It is easily seen that $\operatorname{deg} Q(z) \leq\left(q^{D(l-1)}-1\right) L$ if $Q(z) \neq 0$. Hence, noting that the exponents of the power series of the right-hand side in $(21)$ are greater than $\left(q^{D(l-1)}-1\right) L$ for every $n$, $j$, and $r$, we obtain $b(z)=Q(z)$ and

$$
\sum_{n \geq 0} \sum_{j=1}^{m} \sum_{r=0}^{q^{j}-2}\left(d_{j, r}\left(n+q^{D(l-1)}\right)-d_{j, r}(n)\right) z^{n L+q^{D(l-1)} L-u_{j, r}}=0 .
$$

Define the finite sets

$$
\begin{aligned}
T & =\left\{(j, r) ; 1 \leq j \leq m, 0 \leq r \leq q^{j}-2\right\}, \\
T_{\beta} & =\left\{(j, r) \in T ; r /\left(q^{j}-1\right)=\beta\right\} .
\end{aligned}
$$

Then there exist distinct rational numbers $\beta_{1}, \ldots, \beta_{k}$ such that $T=\bigcup_{i=1}^{k} T_{\beta_{i}}$. In the power series expansion of the left-hand side in (22), if the exponents of $z$ for $(n, j, r)=\left(n_{1}, j_{1}, r_{1}\right)$ and for $\left(n_{2}, j_{2}, r_{2}\right)$ are the same, namely

$$
n_{1} L+q^{D(l-1)} L-u_{j_{1}, r_{1}}=n_{2} L+q^{D(l-1)} L-u_{j_{2}, r_{2}},
$$

then we have

$$
\left|n_{1}-n_{2}\right|=\left|\frac{r_{1}}{q^{j_{1}}-1}-\frac{r_{2}}{q^{j_{2}}-1}\right|<1,
$$


so that $n_{1}=n_{2}$ and $\left(j_{1}, r_{j_{1}}\right),\left(j_{2}, r_{j_{2}}\right) \in T_{\beta_{i}}$ for some $i$. Hence we obtain

$$
\sum_{(j, r) \in T_{\beta_{i}}}\left(d_{j, r}\left(n+q^{D(l-1)}\right)-d_{j, r}(n)\right)=0, \quad n \geq 0
$$

for each $i=1,2, \ldots, k$, that is, the sequence

$$
\left\{\sum_{(j, r) \in T_{\beta_{i}}} d_{j, r}(n)\right\}_{n \geq 0}=\left\{\sum_{(j, r) \in T_{\beta_{i}}} c_{j, r} e_{q^{j}, r}\left(w_{j, r} ; n\right)\right\}_{n \geq 0}
$$

is purely periodic with a period $q^{D(l-1)}$. In particular, noting that $c_{m, r_{m}} \neq 0$, we see that there exist constants $c_{j} \in \boldsymbol{C}$ not all zero and $r_{j}\left(j=1, \ldots, m, r_{j} \in\left\{0, \ldots, q^{j}-2\right\}\right)$ such that

$$
\left\{\sum_{j=1}^{m} c_{j} e_{q^{j}, r_{j}}\left(w_{j, r_{j}} ; n\right)\right\}_{n \geq 0}
$$

is purely periodic with a period $q^{D(l-1)}$. This contradicts Proposition 2.1, and therefore the functions $f_{i, j, r}(z)\left(i=1, \ldots, t, j=1, \ldots, m(i), \quad r=0, \ldots, q^{i}-2\right)$ defined by (14) are algebraically independent over $\boldsymbol{C}(z)$.

The algebraic independence of the values of the functions $f_{i, j, r}(z)$ follows immediately. Indeed, if $f_{i, j, r}(\beta)$ are algebraically dependent over $\boldsymbol{Q}$ for some algebraic number $\beta$ with $0<|\beta|<1$, then by (17) so are the values $F_{i, j, r}(\alpha)$ for $\alpha=\beta^{1 / L}$. This contradicts the algebraic independence of the $F$ 's as we have already proved above, and the proof of Theorem 1.1 is completed.

\section{REFERENCES}

[1] J. P. Allouche And J. Shallit, Automatic sequences: Theory, applications, generalizations, Cambridge University Press, 2003.

[2] K. K. KUвотA, On the algebraic independence of holomorphic solutions of certain functional equations and their values, Math. Ann. 227 (1977), 9-50.

[ 3 ] J. H. Loxton And A. J. VAn Der Poorten, A class of hypertranscendental functions, Aequationes Math. 16 (1977), 93-106.

[4] Yu. V. Nesterenko, Algebraic Independence, Tata Institute of Fundamental Research, New Delhi, 2009.

[ 5 ] K. NishiokA, Mahler functions and transcendence, Lecture Notes in Math. 1631, Springer-Verlag, Berlin, 1996.

[6] K. NishiokA, Algebraic independence of reciprocal sums of binary recurrences II, Monatsh. Math. 136 (2002), 123-141.

[ 7 ] I. Shiokawa ANd Y. Tachiya, Pattern sequences in $\langle q, r\rangle$-numeration systems, Indag. Math. (N.S.) 19 (2008), 151-161.

[ 8 ] I. SHIOKAWA AND Y. TACHIYA, Linear relations between sequences in a $\langle q, r\rangle$-numeration system, Acta Math. Hungar. 132 (2011), 190-206.

[9] Y. TACHIYA, Independence results for pattern sequences in distinct bases, Acta Arith. 142 (2010), 157-167.

[10] Y. UCHIDA, Algebraic independence of the power series defined by blocks of digits, J. Number Theory 78 (1999), 107-118. 
Graduate School of Science and Technology

HIROS AKI UNIVERSITY

HIROS AKI 036-8561

JAPAN

E-mail address: tachiya@cc.hirosaki-u.ac.jp 